Revue à comité de lecture et en accès libre éditée par l'Association Française d'Agronomie sous le numéro ISSN 1775-4240. Plus d'informations www.agronomie.asso.fr/aes

L'AFA est une association à but non lucratif qui publie des travaux en accès libre.

Pour soutenir cette démarche, faites connaître AE\&S, adhérez à l'association et faites adhérer votre organisme et vos collègues!

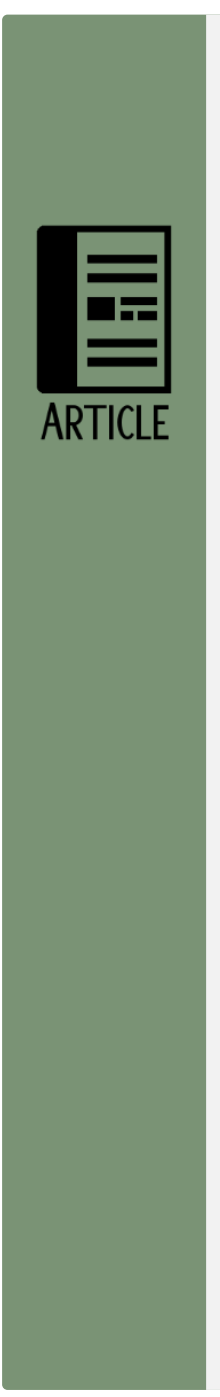

\title{
Une agronomie clinique et territoriale pour accompagner la transition vers une économie circulaire autour de l'agriculture : mise à l'épreuve et enseignements du projet GABiR à La Réunion
}

Vigne $^{a, b}$, M., Achard $c$, P., Alison $c$, C., Castanier ${ }^{d}$, C., Choisis ${ }^{b, e}$, J.P., Conrozier ${ }^{f}$, R., Courdierg, R., Degenne ${ }^{h, i}$, P., Deulvot ${ }^{d}$, A., Dupuy ${ }^{h, i}$, S., Février', A., Hatikg, C., Huatk ${ }^{k}$, J., Kleinpeter ${ }^{a, b}$, V., Kyulavskim $^{m}$, V., Lurette ${ }^{b, n}$, A., Payet ${ }^{\circ}$, A.L., Rondeau $^{p}$, P., Souliéq,r, J.C., Thomas ${ }^{d}$, P., Thuries ${ }^{q, r}$, L., Tillard ${ }^{a, b}$, E., Van de Kerchovef, V., Vayssières $^{a, b}, \mathrm{~J}$.

a CIRAD, UMR Selmet, Saint-Pierre, Réunion, France

b SELMET, Univ Montpellier, CIRAD, INRAE, Institut Agro, Montpellier, France

c Fédération Régionale des Coopératives Agricoles, Saint-Pierre, Réunion, France

d DAAF Réunion, Service Territoire Environnement et Forêt, Saint-Pierre, Réunion, France e INRAE, UMR Selmet, Saint-Pierre, Réunion, France

f Chambre d'Agriculture de la Réunion, Saint-Denis, Réunion, France

g Université de la Réunion, Laboratoire d'Informatique et de Mathématiques, Saint-Denis, Réunion, France

h CIRAD, UMR TETIS, Saint-Pierre, Réunion, France

i TETIS, Univ Montpellier, AgroParisTech, CIRAD, CNRS, INRAE, Montpellier, France. jeRcane, Sainte-Clotilde, Réunion, France k CIRAD, UPR Hortsys, Saint-Pierre, Réunion, France

I HortSys, Univ Montpellier, CIRAD, Montpellier, France $m$ Pôle de Compétitivité Qualitropic, Saint-Denis, Réunion, France $n$ INRAE, UMR Selmet, Montpellier, France o ILEVA, Saint-Pierre, Réunion, France

p Tereos Océan Indien, Sainte-Suzanne, Réunion, France q CIRAD, UPR Recyclage et Risque, Saint-Denis, Réunion, France. r Recyclage et Risque, Univ Montpellier, CIRAD, Montpellier, France Contact auteurs: mathieu.vigne@cirad.fr

\section{Résumé}

Alors que l'économie circulaire (EC) comme nouveau paradigme économique et sociétal émerge, la place du secteur agricole dans les initiatives d'accroissement de la circularité dans les territoires est questionnée. Ce questionnement est d'autant plus pertinent dans un contexte insulaire comme celui de La Réunion, où les enjeux d'autonomie alimentaire et énergétique sont majeurs. De 2017 à 2020, un projet de Recherche et Développement intitulé GABiR (Gestion Agricole des Biomasses sur l'île de La Réunion) a mobilisé des acteurs issus du Développement, de la Formation et de la Recherche, dont de nombreux agronomes de diverses disciplines, mais également des décideurs politiques. Ce projet visait à renforcer l'inclusion du secteur agricole dans l'EC de l'île par une approche territoriale de la gestion des biomasses, valorisées ou valorisables en agriculture. Fort de cette expérience et des acquis du projet, un cadre méthodologique à visée générique relevant d'une démarche clinique à l'échelle territoriale et visant à accompagner la transition vers une EC incluant le secteur agricole est proposé.

Mots clés : Bioéconomie circulaire, Approche territoriale, île de La Réunion, Démarche Clinique 


\begin{abstract}
As circular economy (CE) emerges as a new economic and societal paradigm, the role of agricultural sector to increase circularity in territories is questioned. This questioning is all the more relevant in an insular context like Reunion Island, where food and energy autonomy are major challenges. From 2017 to 2020, a Research and Development project entitled GABIR (Gestion Agricole des Biomasses sur l'île de la Reunion) mobilized actors from Development, Educational Training and Research, including many agronomists from different disciplines, but also policy makers. This project aimed to strengthen the inclusion of agricultural sector in the island's CE through a territorial approach of biomass flows valued or valuable in agriculture. Based on the project experience and achievements, a generic methodological framework, related to a clinical approach at territorial level and aiming to accompany CE transitions, including the agricultural sector, is proposed.
\end{abstract}

Keywords: Circular bioeconomy, Territorial level, Reunion Island, Clinical approach

\title{
Introduction
}

Un nouveau paradigme économique et sociétal prend une place croissante dans les agendas politiques en France (Ministère de la Transition Ecologique, 2018). Il cherche à substituer l'économie dite linéaire, basé sur le principe de 'fin de vie' et d'élimination des déchets, par une vision systémique et multisectorielle dite économie circulaire (EC) favorisant la réduction, la réutilisation, le recyclage et la récupération des matières dans et entre les différents processus économiques (Kirchherr et al., 2017).

L'inclusion du secteur agricole dans cette démarche globale est essentielle. Elle fait écho aux demandes sociétales pour une transition agricole vers notamment plus de sobriété énergétique et une contribution à l'atténuation du changement climatique. C'est ainsi que l'ensemble des acteurs de la Recherche et du Développement, et en particulier les agronomes, est sollicité pour accompagner les initiatives d'EC. Parmi les cadres conceptuels mobilisés, il est souvent fait mention de l'écologie industrielle ou territoriale (Dumont et al., 2013 ; Wassenaar, 2015 ; Malderieux et al., 2017) mais aucune étude ne se prévaut d'une démarche clinique telle que définie par Kockmann et al. (2019).

Partant du principe que sur l'île de la Réunion le secteur agricole, à la fois producteur et utilisateur de biomasses, pourrait tenir un rôle majeur dans ces transitions, un projet de R\&D intitulé « Gestion Agricole des Biomasses sur l'île de la Réunion » (GABiR) a ainsi été mené de 2017 à 2020. Celui-ci visait à renforcer l'inclusion du secteur agricole dans l'EC de l'île par une approche territoriale de la gestion des biomasses.

Cet article vise, dans un premier temps, à expliciter la démarche suivie et le rôle des agronomes dans les différentes étapes du processus. Un focus est ensuite porté sur trois cas d'étude mobilisant la modélisation comme outil de concertation et de co-construction pour illustrer cette démarche. Enfin, à partir des leçons tirées de cette expérience, un cadre générique se prévalant d'une démarche clinique et permettant de résoudre des problématiques en lien avec la gestion territoriale des biomasses est proposé et discuté.

\section{Une approche inclusive en trois étapes}

Le projet GABiR s'est déroulé selon trois étapes (Figure 1): (i) un diagnostic à l'échelle du territoire comprenant les inventaires de la disponibilité des biomasses localement produites et valorisées ou valorisables en agriculture, les gestionnaires de ces biomasses et les problématiques liées à leur gestion, (ii) un processus de sélection de cas d'étude considérés comme emblématiques de la diversité des problématiques locales (diversités technique et logistique, type de biomasses parmi les grands gisements ou encore type de secteurs d'activités de l'île),et (iii) une traitement des cas d'étude sélectionnés. 


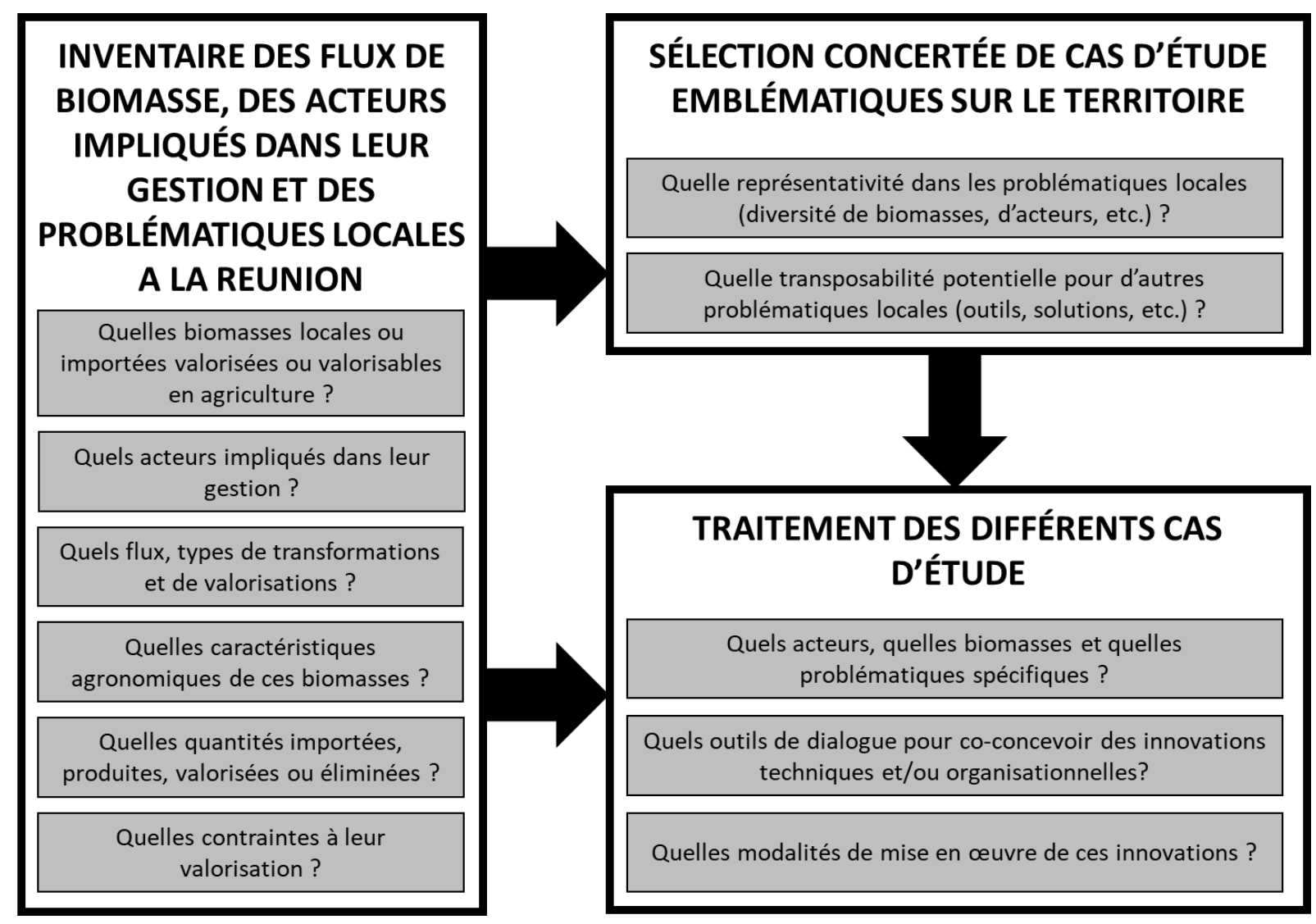

Figure 1. Démarche adoptée dans le projet GABiR, questionnements posés à chaque étape et flux d'information entre les différentes étapes

\section{Diagnostic à l'échelle du territoire basé sur les flux de biomasses}

Ce diagnostic consistait en premier lieu à faire un inventaire et une quantification des flux de biomasses locales valorisées ou valorisables en agriculture, qu'elles soient d'origine agricole ou non-agricole. Pour parvenir à un inventaire le plus exhaustif possible, plusieurs sources de données ont été mobilisées : références bibliographiques, bases de données et entretiens directs (Kleinpeter et al., 2019).

Il est apparu tout d'abord que les gestionnaires des biomasses (personnes physiques ou morales) sont issus de 7 secteurs (Figure 2).

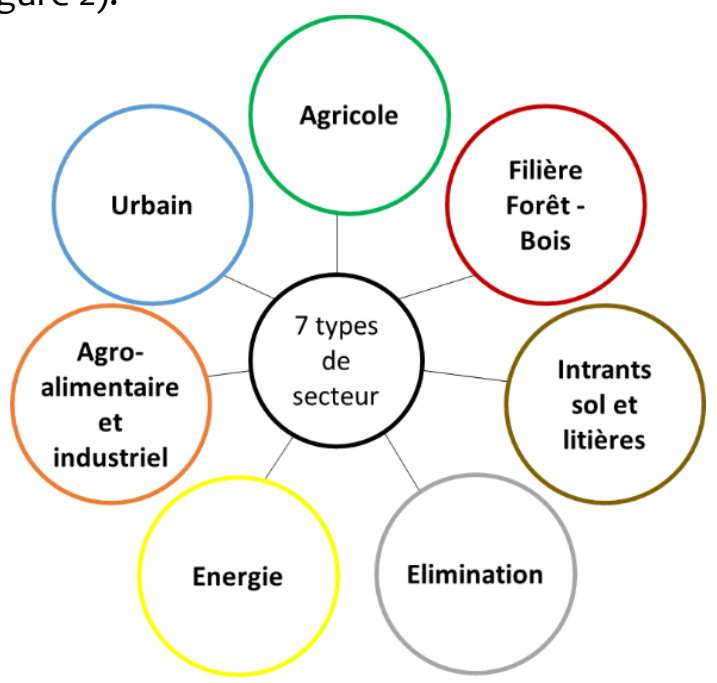

Figure 2. Types de secteur d'origine des biomasses recensées dans le projet GABiR 
L'ensemble de la population réunionnaise (environ 853.000 habitants recensés en 2016 selon I'INSEE) a été considéré comme producteur de biodéchets ménagers. A ceux-ci, s'ajoute environ 8.000 autres producteurs et/ou utilisateurs de biomasses, dont près de 7500 exploitants agricoles. De plus, 107 biomasses différentes ont été inventoriées. Elles ont été regroupées en 11 catégories telles que les effluents d'élevage, les co-produits et déchets issus du secteur agroalimentaires et industriels (AAI), y compris la filière sucre ou encore les biomasses issues du secteur urbain (boues de stations d'épuration, biodéchets issus des déchets ménagers et assimilés, etc.).

Enfin la quantification des différents types de biomasse, de leur secteur d'origine et de leur valorisation (Figure 3) a montré que la valorisation principale de ces biomasses se fait en agriculture (alimentation et litière animale, engrais organique et amendement, support de culture type terreau, paillage des sols).

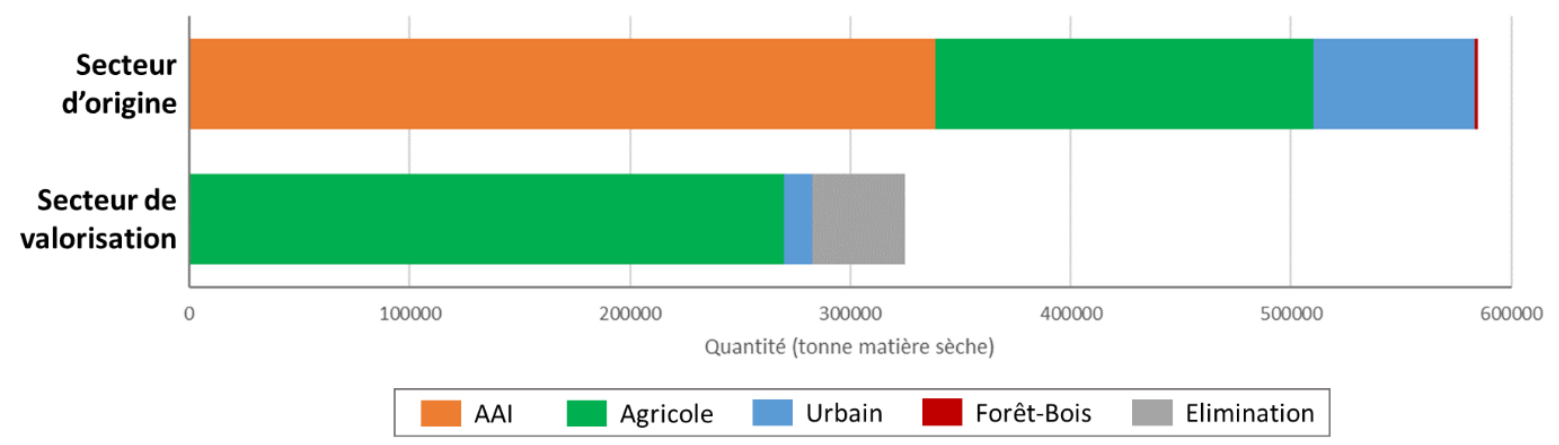

Figure 3. Quantification (en tonne de matière sèche) des flux selon le secteur d'origine et de valorisation des biomasses valorisées ou valorisables en agriculture sur l’île de la Réunion

\section{Une sélection de cas d'étude emblématiques}

Ce diagnostic a également permis une large consultation de l'ensemble des acteurs concernés par les biomasses locales ou importées et valorisées ou valorisables en agriculture. Ces derniers ont ainsi pu lister différents problèmes et contraintes liés à la gestion des biomasses et parfois émettre des premières pistes de solutions à mettre en place. En accord avec ces derniers, ces problématiques ont été formalisées sous forme de fiches qui ont donné lieu à des propositions de cas d'étude à destination du projet et ont été soumis au collectif partenarial. Parmi la vingtaine de propositions émises, l'ensemble des partenaires a ainsi fait le choix concerté de retenir cinq cas d'étude qui ont fait l'objet de groupe de travail ad hoc et ont été investigués par la suite :

- La mise en place d'une filière de fourrages structurée, facilitant le transfert de fourrages conservés entre exploitations à l'échelle de l'île ;

- La recherche de leviers permettant de réduire les contraintes d'épandage des effluents d'élevage dans la commune de Saint-Joseph,

- L'évaluation de l'intérêt du co-compostage d'effluents d'élevage et de déchets verts pour pallier aux contraintes d'épandage des élevages monogastriques et d'évacuation des déchets verts sur les plateformes des Syndicats Mixtes de Traitement des Déchets (SMTD),

- L'évaluation du potentiel de valorisation agricole des biodéchets,

- La caractérisation des besoins en biomasses et les contraintes liées à leur gestion dans les exploitations en Agriculture Biologique.

Ce choix a été motivé par plusieurs critères : (i) l'importance (occurrence, volumes produits) des biomasses dans l'inventaire précédemment réalisé, (ii) le nombre et le type d'acteurs impliqués, y compris hors du secteur agricole, (iii) leur valeur heuristique et leur capacité à aboutir à des outils et des solutions mobilisables sur d'autres parties du territoire, (iv) la contribution potentielle de la résolution de la problématique pour améliorer l'autonomie du secteur agricole et du territoire et (v) la capacité à mobiliser les compétences internes au projet, en particulier en sciences agronomiques, pour la résolution de ces problématiques. 
Comme ils ont mobilisé la modélisation comme outil de concertation et de co-construction et abouti à des résultats mobilisés par les acteurs locaux, seuls les trois premiers cas d'étude listés précédemment sont présentés dans cet article

\section{Du diagnostic à l'identification de solutions collectives}

Mise en place d'une filière de fourrages structurée à l'échelle de l'île de la Réunion

On observe régulièrement sur l'île de la Réunion des déficits fourragers au niveau des élevages en hiver austral, plus particulièrement durant les années plus sèches, pouvant conduire à l'importation ponctuelle de fourrages. Par ailleurs, on observe une répartition spatiale inégale du potentiel fourrager entre zones d'élevage de ruminants (e.g. Hauts de l'Ouest peu arrosés vs zones des Plaines plus humides) liée à une grande diversité de microclimats.

Alors que le potentiel fourrager global annuel semble suffisant pour permettre l'autonomie fourragère de l'ensemble des filières de ruminants (BRLi, 2017), une question, portée par I'Association Réunionnaise de Pastoralisme (ARP) en partenariat avec les filières de ruminants, était la suivante : quelles seraient les modalités de mise en œuvre d'une filière fourrage structurée à l'échelle de l'île de la Réunion ? L'objectif étant de réduire la dépendance des exploitations et de l'île aux produits d'importation, tels que les concentrés, pour le maintien et la viabilité de ses activités d'élevage.

Des travaux de quantification et localisation des productions et des consommations de fourrages réalisées à partir d'une cartographie précise des parcelles fourragères et une géolocalisation des troupeaux de ruminants (Lorre, 2019 ; Magnier, 2019) ont abouti notamment aux calculs des balances fourragères annuelles à l'échelle de 12 zones de production tenant compte de l'altitude, du relief et du réseau routier (Figure 4).
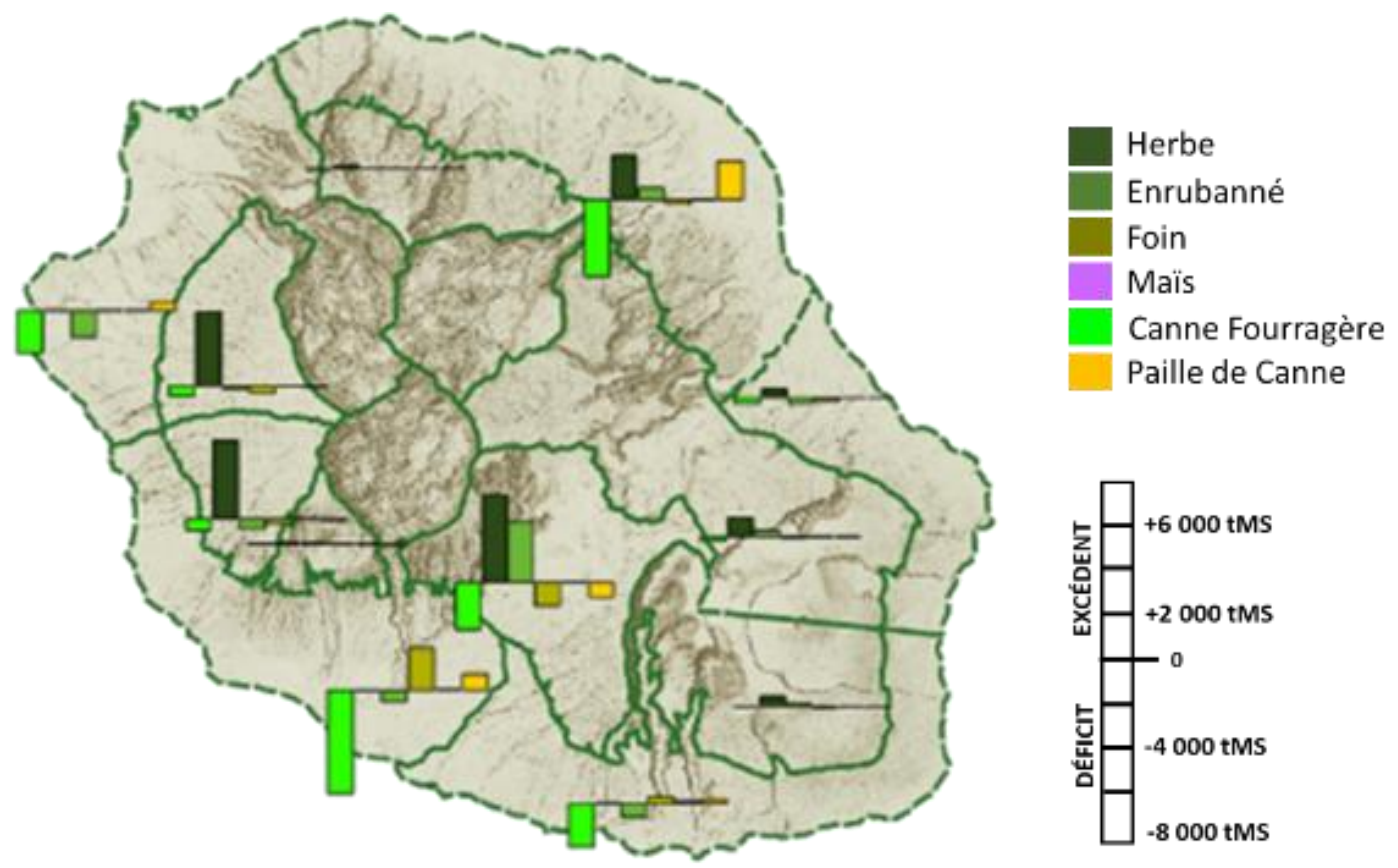

Figure 4. Balances fourragères annuelles (en tonnes de matière sèche par an) dans 12 zones logistiques de l’île de la Réunion (Lorre, 2019)

Les balances obtenues montrent des déséquilibres entre différentes zones. Faisant l'hypothèse que des transferts de fourrages entre zones permettraient un rééquilibre de ces balances fourragères, trois scénarios favorisant les échanges de fourrages ont été co-construits en partenariat avec les professionnels de l'élevage réunionnais :

- Scénario de référence So : représentation au mieux de la réalité des transferts actuels ;

- Scénario S1: Mise en place d'une filière fourrage structurée se traduisant par la présence 
de 4 structures de stockage à différents endroits du territoire ;

- Scénario S2: S1 + mise en œuvre de leviers techniques (récupération de friches, fauche des parcelles jusque-là uniquement pâturées et amélioration de la gestion du pâturage) visant l'augmentation des stocks réalisés en période favorable dans certains élevages.

Ceux-ci ont été représentés puis évalués selon plusieurs indicateurs (volumes de fourrages échangés ou encore nombre d'élevages impliqués) sous Ocelet ${ }^{\circledR}$ (Degenne et al., 2016). Il est apparu que le scénario S2 entraîne une augmentation de la proportion d'élevages dont les besoins sont couverts en période hivernale (Lorre et al., 2020) en dépit d'une distance totale à parcourir plus élevée (près de $20000 \mathrm{~km}$ supplémentaires par rapport à So et $\mathrm{S} 1$ ). Ces résultats alimentent actuellement une réflexion multi-acteurs sur la mise en place d'une filière fourrage structurée et de ses différentes modalités.

Identification des contraintes majeures à l'épandage des effluents d'élevage sur l'île de la Réunion Dans la commune de Saint-Joseph (près de $178 \mathrm{~km}^{2}$ dont environ 3000 ha de surfaces agricoles), les difficultés d'épandage des effluents sont un frein majeur au développement des activités d'élevage. Des enquêtes réalisées auprès d'éleveurs de la zone démontrent que les dynamiques d'urbanisation qui réduisent les surfaces agricoles devenues constructibles sont une contrainte majeure, d'autant qu'elles exacerbent les contraintes réglementaires (distance aux habitations) déjà prégnantes du fait des caractéristiques physiques de la zone (distances aux cours d'eau, fortes pentes et risque de ruissellement).

Deux questions portées par les parties prenantes de la problématique (éleveurs, représentants de la commune et instituts techniques) ont ainsi été posées : Les éleveurs ont-ils une marge de manœuvre concernant les surfaces disponibles pour l'épandage, ou le territoire est-il déjà saturé compte-tenu des obligations réglementaires ? Est-il possible de couvrir les besoins des cultures par de la fertilisation organique produite localement?

Grâce à un travail important d'inventaire et d'analyse de données (cartographie de l'occupation du sol, recensement des exploitations agricoles et des plans d'épandages), deux outils ont été développés sous Ocelet ${ }^{\circledR}$ (Degenne et al., 2016).

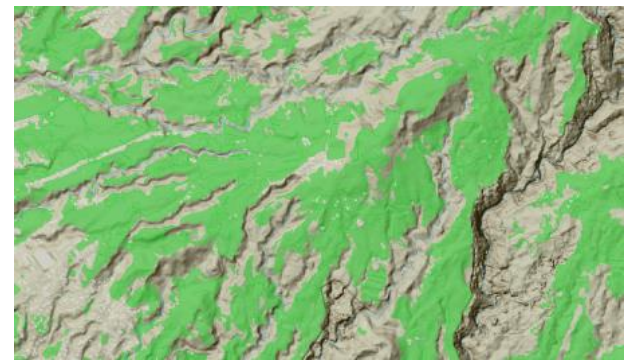

(a)

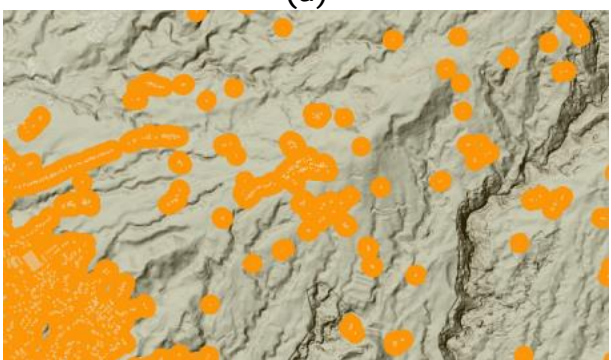

(c)

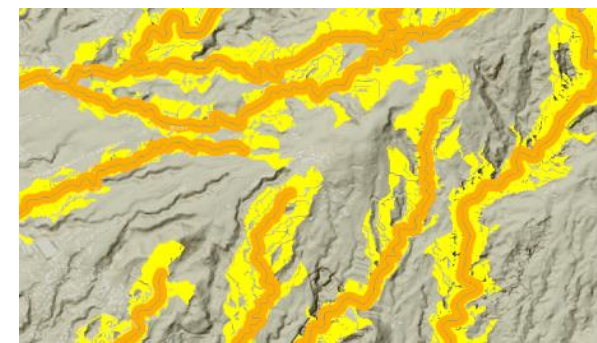

(b)

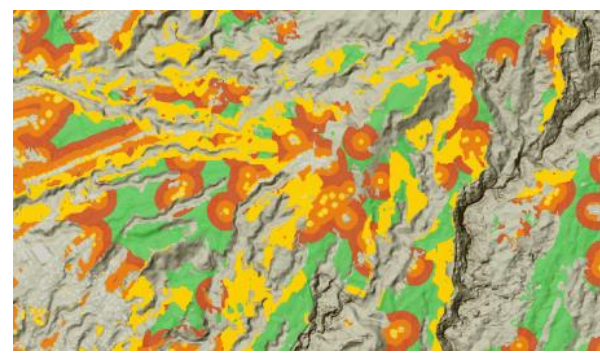

(d)

Figure 5. Exemple des différentes étapes de la cartographie des surfaces épandables sur la commune de Saint-Joseph : (a) représentation du parcellaire, (b) caractérisation de bandes de 35m de part et d'autre des cours d'eau (orange) et estimation des risques de ruissellement par le logiciel (en jaune), (c) représentation de zones situées à $50 \mathrm{~m}$ du bâti illustrant les zones d'exclusion d'épandage pour du fumier et (d) découpage des zones non épandables sur le parcellaire fourni et assemblage sous forme de carte (chaque couleur indiquant des contraintes de différentes natures) 
Le premier est un logiciel permettant d'estimer les surfaces épandables à l'échelle de la commune. Il s'est appuyé sur le parcellaire cultivé obtenu par télédétection (Dupuy et al., 2020), un modèle numérique de terrain représentant la topographie de la commune et les différents aspects réglementaires (distance aux cours d'eau, aux points de captage, au bâti et risque de ruissellement) en lien avec le type de matière épandue et le régime règlementaire (Règlement Sanitaire Départemental ou Installation Classée Protection de l'Environnement) (Figure 5).

Les surfaces épandables totales, à l'échelle de la commune, calculées selon des contraintes fixes de distance aux cours d'eau et de risques de ruissellement mais variables selon la distance au bâti sont reportées dans le Tableau 1.

Tableau 1. Estimation de la proportion de surfaces épandables (en \% des surfaces agricoles totales) dans la commune de Saint-Joseph sous différentes contraintes de distance d'épandage aux habitations

\begin{tabular}{lccc}
\hline \multirow{2}{*}{ Type de cultures } & \multicolumn{2}{c}{ Distance d'épandage minimum aux habitations } \\
\cline { 2 - 4 } & $\mathbf{1 0 0}$ & $\mathbf{5 0 m}$ & $\mathbf{1 0 m}$ \\
\hline Maraichage & $0^{\mathrm{a}}$ & 47 & 93 \\
Arboriculture & 29 & 56 & 93 \\
Prairies $^{\mathrm{b}}$ & 49 & 76 & 97 \\
Canne à sucre & 47 & 73 & 98 \\
Total & 29 & 58 & 94 \\
\hline
\end{tabular}

a La limitation d'épandage à moins de 100 mètres d'une habitation porte sur le lisier. Ce dernier étant interdit sur les cultures maraichères, la surface épandable est donc nulle.

b Prairies de fauche

On observe que lorsque la matière épandue peut l'être à $10 \mathrm{~m}$ des habitations (cas du compost), une grande partie des parcelles cultivées restent épandables (94\%). Par contre, lorsque la réglementation impose un épandage à plus de $100 \mathrm{~m}$ des habitations (cas des lisiers non traités), les surfaces épandables sont largement réduites ( $29 \%$ des surfaces en arboriculture et près de la moitié des surfaces de prairies et en canne à sucre). (eci confirme que les contraintes réglementaires, en particulier en lien avec la proximité du bâti des surfaces agricoles, sont majeures dans le cas des effluents traditionnellement produits dans les élevages de la zone (fumier de bovins ou de volailles, lisier de porcs).

Mobilisant le parcellaire cultivé mais également les plans d'épandage déclarés par les agriculteurs de la commune, le second outil a permis d'estimer et cartographier un potentiel théorique de la couverture des besoins azotés des différentes parcelles par les effluents d'élevage épandus (Fig 6).

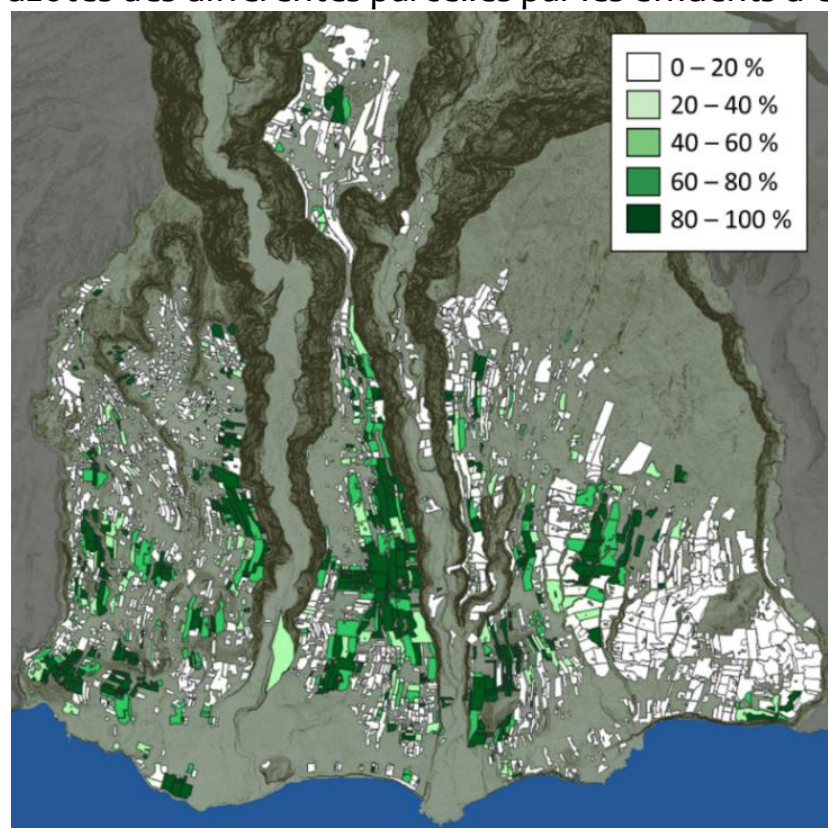

Figure 6. Taux de couverture des besoins en azote organique des différentes parcelles de la commune de Saint-Joseph par les apports d'effluents d'élevage estimés à l'aide des plans d'épandage (Jarry, 2019) 
Les résultats obtenus montrent qu'une proportion importante des surfaces du territoire ne reçoit pas ou peu de fertilisants organiques. A l'échelle de la commune, ces derniers couvrent seulement $30 \%$ des besoins en azote des cultures. Ce chiffre recouvre toutefois une forte hétérogénéité. Dans les quartiers Est, dominés par la canne à sucre et avec peu d'élevages, ce taux de couverture est faible voire nul. A l'inverse, les parcelles proches des bâtiments d'élevage affichent des taux de couverture pour la plupart supérieurs à $80 \%$. Ces résultats confirment également l'importance des contraintes logistiques, principalement le coût du transport d'effluents sur de longues distances, qui tend à concentrer la recherche de surfaces épandables dans les mêmes zones (proches des zones de production des effluents).

Plusieurs pistes permettant de réduire ces deux types de contraintes, réglementaires et logistiques, ont été discutées par les parties prenantes. La transformation des effluents, en particulier sous forme de composts, est ainsi envisagée. Elle permettra de diminuer la pression sur les parcelles à proximité des élevages en augmentant la surface épandable mais également en facilitant le transport de la matière sur des zones plus éloignées. La construction prévue d'une plateforme de traitement des déchets verts dans la commune pourrait notamment représenter une opportunité intéressante pour transformer ces effluents à l'image du cas d'étude suivant.

\section{Valorisation en agriculture des déchets verts urbains dans le sud de l'île de la Réunion}

Sur l'île de la Réunion, les particularités climatiques (climat tropical) et culturelles (importance des jardins en milieu urbain) entraînent une production de déchets verts supérieure à la moyenne nationale (Cornec et Mir, 2018) dont la gestion est parfois problématique pour les collectivités. Les gestionnaires de la plateforme de traitement de la Rivière Saint-Etienne par exemple, dans le SudOuest de l'île, font ainsi face à des phénomènes de saturation sur certaines périodes, notamment en saison des pluies. Dans la même zone, certains éleveurs de monogastriques rencontrent des difficultés à gérer l'épandage de leurs lisiers sur lesquels une règlementation stricte s'applique comme mentionnée dans le cas d'étude précédent.

Des premiers éléments issus de projets antérieurs tels que Girovar (MAAP, 2015 ; Wassenaar et al., 2015), complétés pendant la première phase de diagnostic général du projet (Gaffier, 2017), suggéraient l'utilisation de déchets verts en co-compostage avec ces effluents d'élevage permettant un changement de statut règlementaire de ces derniers, qui passent ainsi du statut de " déchet » à celui d'un produit normé. Ce changement présente un double intérêt : il permet aux éleveurs de se dispenser de plan d'épandage et aux maraichers de disposer de matières organiques dont les critères qualitatifs sont encadrés par des normes.

C'est ainsi qu'lleva, SMTD de l'ouest et du sud de l'île, et des représentants des éleveurs de la zone, ont porté conjointement une question auprès des partenaires du projet : quelles seraient les modalités économiques et logistiques pour la mise en place d'une filière de co-compostage dans la zone?

Après un diagnostic des intérêts, des besoins et des contraintes de chaque acteur, y compris des maraichers, utilisateurs potentiels du compost produit mais qui faisaient preuve jusque-là d'une méfiance sur la qualité sanitaire des biomasses, des solutions représentées par différents scénarios d'organisation ont ainsi été étudiés par modélisation (Darras, 2019) (Figure 7) :

- un co-compostage individuel à la ferme, chez les éleveurs,

- un co-compostage individuel à la ferme, chez les maraichers utilisateurs du produit final,

- la création de plateformes collectives de co-compostage, de taille modeste, gérées par des groupements de 5 à 10 éleveurs,

- et la mise en place d'une plateforme collective de co-compostage pour l'ensemble des éleveurs de la zone. 


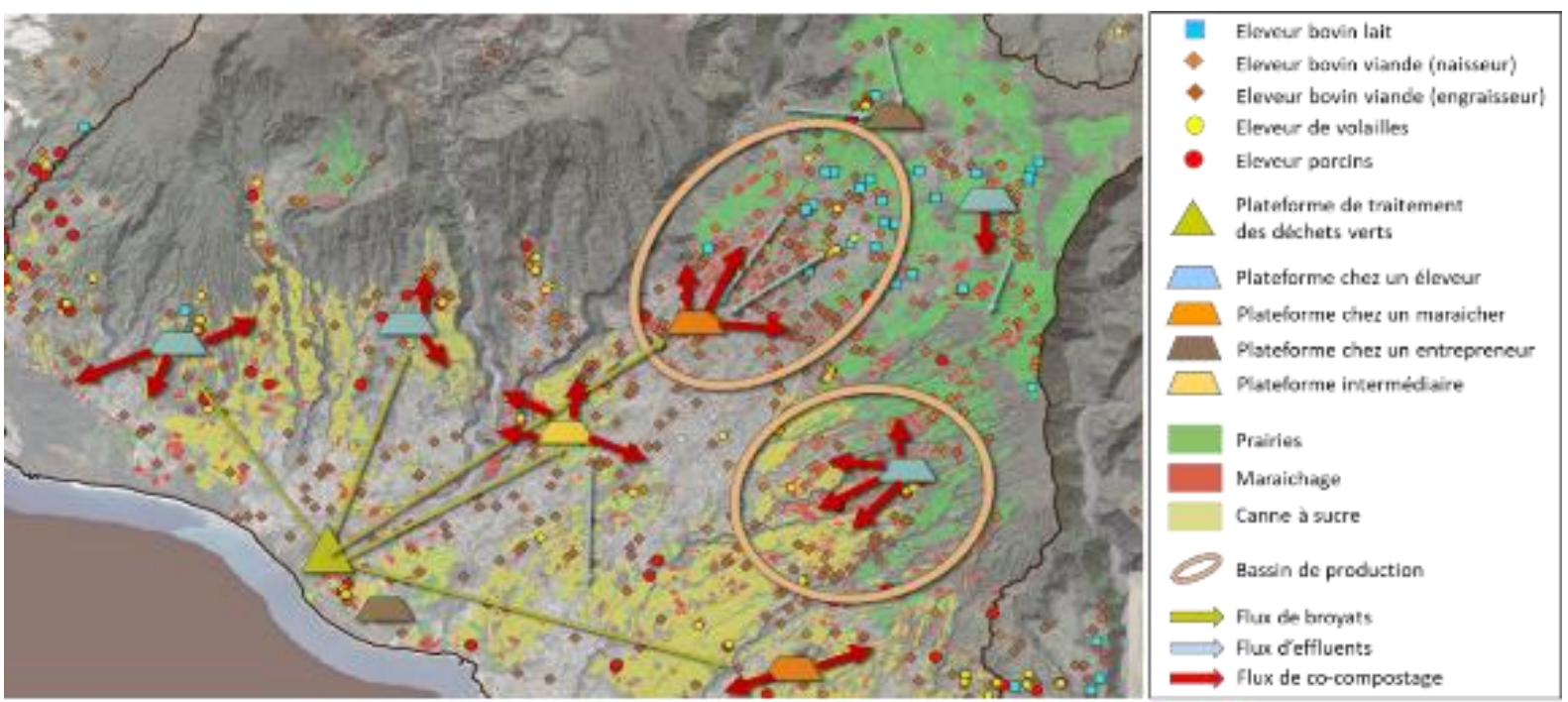

Figure 7. Représentation schématique des différents scénarios dans la zone d'étude (Darras 2019)

Ces scénarios ont été représentés à l'aide de l'outil GAMA® (Taillandier et al., 2018) et évalués d'un point de vue socio-technique (e.g. besoins en différentes matières pour un mélange adapté aux besoins des maraichers, temps de travail, compétences nécessaires) et économique (e.g. kilomètres parcourus par les différents acteurs, besoins d'investissement). La mise en discussion des résultats obtenus avec différentes parties prenantes potentiellement impliqués dans la filière a abouti au constat qu'il semblait plus judicieux d'envisager une combinaison de scénarios comptetenu des diverses situations dans les sous-zones qui la composent. Néanmoins, la dynamique actuelle, portée principalement par les éleveurs de monogastriques, se dirige vers plusieurs projets de plateformes en exploitation positionnées à proximité des élevages voire directement dans les élevages, du fait des contraintes de transport des effluents et des réticences à la mise en place d'une plateforme collective.

\section{Quelle approche clinique à l'échelle territoriale?}

Si selon Kockmann et al. (2019), les démarches cliniques ont a priori leur pertinence à l'échelle territoriale, devenue prégnante dans le développement, elles semblent avoir été peu mobilisées à cette échelle. Pourtant, les grands principes qui guident l'approche clinique (les relations entre acteurs, la posture d'intervention, le processus dynamique basé sur l'observation et le raisonnement) sont mobilisables pour traiter des problématiques de mise en place d'une EC à l'échelle territoriale, tout comme le diagnostic partagé, la mise en discussion et l'identification conjointe de solutions. Cependant, l'expérience menée dans le cadre du projet GABiR à l'échelle de l'île de la Réunion permet d'identifier des spécificités propres à cette échelle qui pourrait venir compléter ces grands principes.

\section{Un diagnostic multi-échelle et multi-acteur au cœur de la démarche}

Selon Cerf et al. (2019), « le diagnostic agronomique, particulièrement lorsque l'action doit se situer dans une stratégie long terme, est l'étape essentielle de la démarche clinique ». A l'échelle d'un territoire, ce diagnostic est d'autant plus difficile à mener qu'il traite d'une multitude d'objets, reliés à de nombreux acteurs porteurs d'une diversité de problématiques. Plusieurs cadres couplant l'analyse spatiale et organisationnelle ont déjà été proposés pour appréhender la complexité de cette échelle d'analyse (Moine, 2006 ; Leloup, 2010).

Conscients de cette complexité, nous avons organisé la démarche de diagnostic autour de deux grandes étapes : une première à l'échelle de l'île de la Réunion et une seconde spécifique à chaque cas d'étude. Le premier diagnostic visait à caractériser les enjeux et les problématiques prioritaires de l'île, à partir d'une vision la plus exhaustive possible, des acteurs et biomasses en jeu. Le second 
était un préalable à la recherche d'innovations dans le pilotage du système tel que décrit par Cerf et al. (2019). Il s'inscrivait dans une démarche de recherche-action (Vall et Chia, 2014 ; DellaCroce et Tironi, 2018), et visait à comprendre les processus en jeu, les comportements des acteurs et les contraintes spécifiques à chacun.

Toutefois, ces deux étapes étaient intimement liées. Au-delà de la caractérisation des problématiques majeures de gestion des biomasses d'origine agricole et/ou valorisables en agriculture, la première étape a ainsi permis (i) de produire des pré-diagnostics propres à chaque cas d'étude (quels acteurs ? quels freins ? quelles pistes de leviers déjà engagés ou envisagés ?) et (ii) de fournir des données qualitatives et quantitatives facilitant la seconde étape de diagnostic approfondi et la mobilisation d'outils cartographique et de modélisation.

Par ailleurs, un point commun majeur à ces deux étapes réside dans l'approche participative orientée multi-acteurs. Cette mobilisation a favorisé les synergies entre parties prenantes et la construction de projets collectifs faisant consensus. Enfin, la mobilisation de représentants des différents types d'acteurs permet d'intégrer le contexte institutionnel et politique et d'aborder les questions de gouvernance en lien avec la thématique visée. Dans le cas du projet GABiR, l'association des institutions en charge des politiques publiques (Région, Département, Commune, DAAF, DEAL) a permis, grâce au dialogue, de donner à voir à ces acteurs les réalités et contraintes locales et d'appréhender la latitude d'actions de ces derniers dans un contexte réglementaire établi à l'échelle nationale.

\section{Une relation entre des acteurs d'origine variée}

Prévost et al. (2017) définissent l'agronomie clinique comme " l'agronomie qui étudie l'agroécosystème en vue d'établir un diagnostic agronomique de son fonctionnement pour proposer des améliorations correspondant aux compromis souhaités par l'agriculteur ». L'agronome est ainsi en accompagnement de «l'agriculteur-praticien, confronté à des décisions tactiques ou stratégiques » (Kockmann et al., 2019).

Dans les problématiques de bioéconomie circulaire, l'échelle d'analyse et la diversité des acteurs impliqués élargissent l'application de la démarche clinique à un collectif aux compétences, objectifs et intérêts variés et ne la limite pas à la relation agronome-praticien. Le premier cas d'étude du projet GABiR, qui concerne la mise en place d'une filière fourrages, fait intervenir trois groupes d'acteurs : les agronomes-chercheurs (zootechniciens et modélisateurs), les éleveurs et l'encadrement technique représenté par des structures diverses selon les filières d'élevage étudiées (ccopératives, Chambre d'Agriculture, Association Réunionnaise de Pastoralisme). Dans les deux autres cas d'étude, le collectif est élargi à des représentants des services de l'Etat spécialistes de la réglementation sur la gestion des biomasses, des collectivités territoriales en charge des politiques locales agricoles et urbaines et des gestionnaires de plateformes de traitement des déchets.

Le rôle de l'agronome dans la réalisation du diagnostic puis en appui à la construction de scénarios pour l'aide à la décision se complexifie alors. Il s'agit de prendre en compte la diversité des connaissances mais également d'appréhender les représentations et stratégies de chaque acteur. Pour exemple, la spécificité des gestionnaires de plateformes de traitement des déchets réside dans leur interface entre société civile (au service des habitants via la gestion de leurs déchets), secteurs économiques dont agricole en particulier (via la mise à disposition d'une matière pour leur fonctionnement), et monde politique (de par leur dépendance des stratégies d'aménagement du territoire décidés par les élus). Il devient donc nécessaire de construire des arènes de concertation où l'agronome devient « médiateur », tel que décrit par Sebillotte (2002, 2006). 


\section{La modélisation comme outil de diagnostic et de concertation facilitant l'émergence d'innovations territoriales}

Dans le projet GABiR, un choix primordial a été de mobiliser la modélisation comme outil de représentation, même simplifiée, des processus pour chaque cas d'étude. Utilisé comme objet intermédiaire (Vinck, 1999; Lardon, 2005), l'outil a fortement favorisé l'appropriation par les parties prenantes des problématiques mises en lumière lors des phases de diagnostic. En effet, comme illustré précédemment dans les cas d'étude, un diagnostic préliminaire est souvent posé par les acteurs du territoire face à une problématique rencontrée. Or, les hypothèses émises par ceux-ci sur les causes des problèmes et les freins aux solutions potentielles peuvent être antagonistes. Comme illustré précédemment, c'est le cas par exemple à Saint-Joseph où la forte contrainte ressentie par les éleveurs, liée au phénomène d'urbanisation, s'est révélée en réalité limitée grâce à la représentation permise par les outils de modélisation.

Cet état de fait rend parfois impossible la mise en place d'un " diagnostic-pronostic partagé » (Kockmann et al., 2019). Il est alors du rôle de l'agronome d'élaborer un diagnostic basé sur des données étayées. Alors qu'elles sont difficiles à établir sur des échelles d'étude aussi larges, la modélisation semble particulièrement intéressante pour les acquérir et ainsi construire ce diagnostic partagé. La formalisation des connaissances sous forme de modèles favorise l'intégration des points de vue de différentes parties prenantes d'un territoire et aide chacun à mieux appréhender son rôle par rapport aux autres dans le système complexe que ce territoire constitue.

Par ailleurs, la mobilisation d'outils cartographiques et de simulation-exploration spatialement explicites s'avère féconde pour représenter des scénarios de flux de biomasses sur un territoire et favoriser l'émergence de solutions collectivement partagées. Cet outil est particulièrement utile quand les processus n'obéissent pas seulement à des considérations techniques ou financières. Dans le cas d'étude de Saint-Joseph, par exemple, les stratégies d'épandage sont fortement dépendantes de réseaux sociaux informels qui construisent les couples producteurs / consommateurs d'effluents. La modélisation permet de simuler des appariements producteurs / consommateurs offrant une meilleure couverture des besoins, de part et d'autre, et tenant compte des contraintes logistiques et règlementaires. Ces résultats peuvent ensuite être mobilisés pour objectiver un potentiel d'amélioration basé sur une évolution de ces réseaux d'échange, en favorisant l'interconnaissance et la mise en lumière d'intérêts communs.

\section{Du cas d'étude à une stratégie régionale en faveur de l'économie circulaire sur l'île}

L'approche clinique mobilisée dans le projet, dont les cas d'étude constituaient un élément majeur, était motivée par la nécessité d'avoir une vision pragmatique de la complexité du système étudié qu'est l'île de la Réunion. On doit s'interroger toutefois sur la valeur heuristique des cas d'études et la proposition de solutions à l'échelle de problématiques locales, pour élaborer un projet territorial d'économie circulaire à l'échelle de l'île. Bien que ce changement d'échelle n'ait pas été réalisé dans le projet, le pari est que les connaissances générées dans les cas d'étude ont une valeur générique qui les rendent remobilisables pour appuyer les acteurs locaux. Cette généricité nous semble se fonder, a minima, sur plusieurs prérequis.

En premier lieu, il convient de s'assurer de la reproductibilité des démarches et des solutions proposées sur les problématiques voisines dans d'autres contextes de l'île. L'étape clé est ici la phase de sélection des cas d'études qui doit s'assurer de la représentativité de ces derniers au regard du diagnostic territorial global. Cela signifie que les solutions proposées et mises en œuvre pourront avoir un impact majeur sur l'économie circulaire du territoire car les volumes de biomasses cibles sont proportionnellement importants ou parce qu'ils sont représentatifs de problématiques similaires ailleurs sur le territoire. Par ailleurs, il convient également d'orienter les sorties des outils en faveur de la concertation et de la co-construction de solutions innovantes. Dans le cadre du projet GABiR, ces outils, principalement de cartographie et de modélisation, ont montré leur capacité à appréhender des problématiques diverses, voire à mener des analyses à l'échelle de 
l'île comme la cartographie de l'occupation des sols (Dupuy, 2020) ou l'estimation des surfaces épandables (Jarry, 2019). Ensuite, pour que ces outils soient vecteurs de changement, il semble nécessaire que le collectif du projet soit constitué de représentants de l'ensemble des filières agricoles.

De plus, il y a synergie lorsque chaque partenaire est au même niveau d'information et que le collectif est à même d'appréhender les problématiques et leur résolution collective. Pour faciliter la transmission des informations, mais également s'assurer de l'homogénéité des démarches, il apparait nécessaire que tout ou une partie importante de ce collectif soit investi sur l'ensemble des cas d'étude. Le traitement des cas d'étude par des collectifs indépendants conduit, en effet, à un risque de cloisonnement qui peut constituer un frein au changement d'échelle pour les mêmes raisons qu'exposées précédemment. Ce collectif transversal doit assurer l'articulation entre les échelles micro, méso et macro (respectivement les échelles des cas d'étude et l'échelle de l'île dans le cas de GABiR).

Toutefois, cette stratégie globale ne peut pas s'appuyer sur un ensemble de solutions localisées, issues des cas d'étude du projet ou élaborées à partir des outils construits. Il est nécessaire de mieux prendre en compte la multiplicité des enjeux territoriaux, particulièrement divers dans le cas d'un territoire insulaire, les effets de synergies et complémentarités entre secteurs et territoires de l'île mais également les effets de compétition. Elle peut s'appuyer sur des compromis à construire entre les différents enjeux du territoire, tel que l'augmentation conjointe de l'autonomie alimentaire et de l'autonomie énergétique sachant que des biomasses locales, comme les produits issus de la canne à sucre trouvent leur utilité aussi bien dans les secteurs agricoles qu'énergétiques.

\section{Vers un cadre générique pour une démarche clinique à l'échelle territoriale}

Bien que le changement d'échelle des résultats des cas d'étude pour la construction d'innovations à l'échelle de l'île n'a pu être effectué, les acquis et retours d'expérience permettent d'esquisser les premiers contours d'un cadre générique pour la mise en place d'un projet territorial d'économie circulaire, s'appuyant notamment sur le secteur agricole, et qui relèverait d'une démarche clinique (Figure 8).

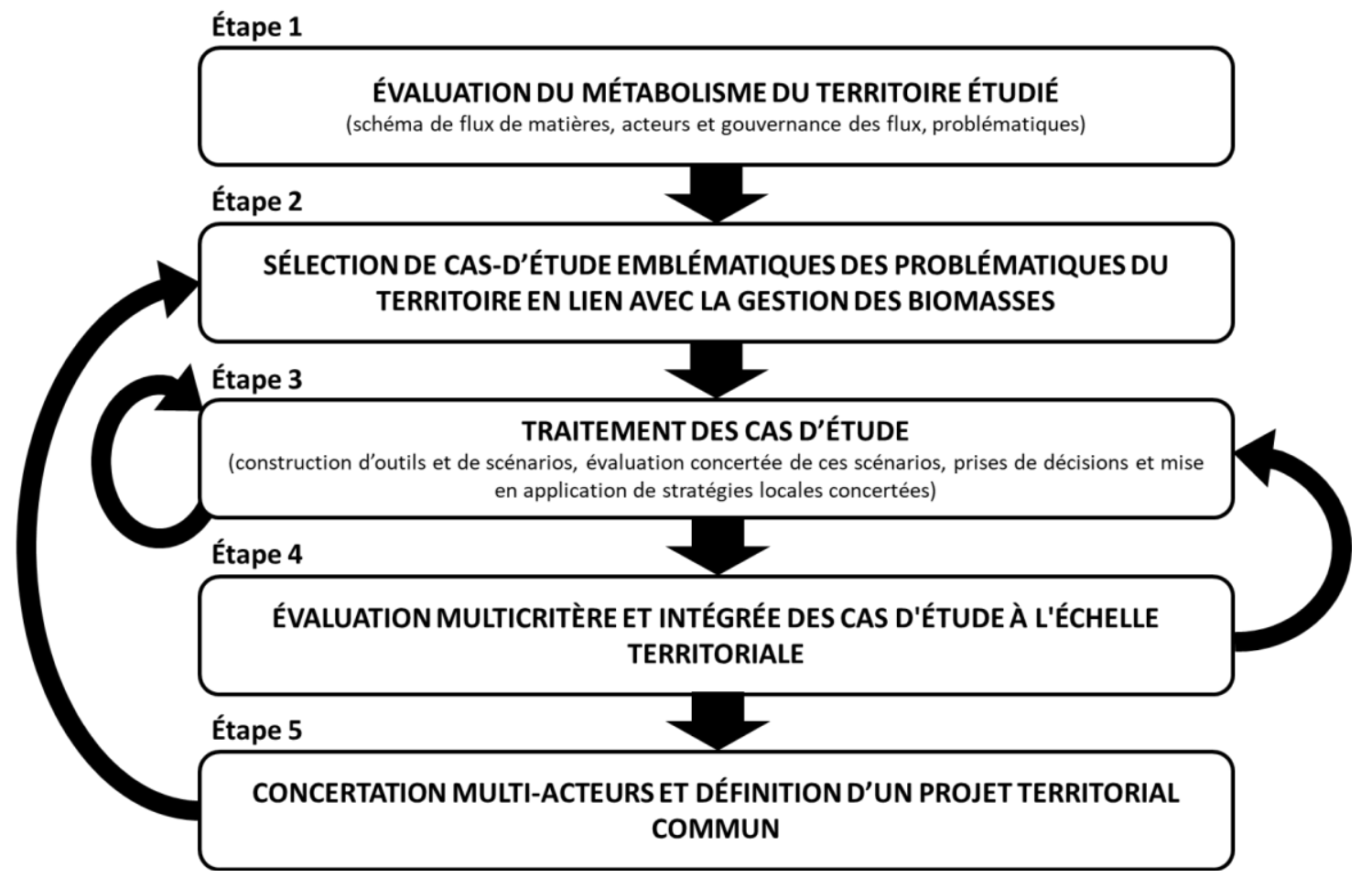

Figure 8. Proposition d'un cadre générique pour l'élaboration d'un projet territorial d'économe circulaire favorisant l'inclusion du secteur agricole 
Ce cadre, s'inspirant largement de la démarche du projet GABiR, pourrait ainsi s'appuyer sur 5 étapes successives :

(i) L'évaluation du protométabolisme (stock et flux de matières et d'énergie) des matières utilisés et utilisables en agriculture sur le territoire étudié. Cette étape, même si elle peut être consommatrice de temps comme ce fût le cas pour le projet GABiR, est indispensable et doit aboutir à un schéma de flux de matières à l'échelle territoriale mais également à un inventaire le plus exhaustif possible des acteurs impliqués dans la gestion de ces flux et des problématiques locales rencontrées. La démarche de métabolisme territorial semble pertinente pour atteindre cet objectif (Bonaudo et al., 2016) en aboutissant ainsi à la définition du protométabolisme du territoire étudié (Grillot et al., 2021).

(ii) Une sélection de cas d'études emblématiques, à des échelles inférieures du territoire étudié, des problématiques identifiées dans l'étape 1. Comme ce fût le cas dans GABiR, cette sélection devrait s'appuyer sur cinq critères principaux : (a) la part des biomasses du cas d'étude dans le protométabolisme du territoire étudié, (b) le nombre et le type d'acteurs impliqués, (c) leur capacité à aboutir à des outils et des solutions mobilisables sur d'autres parties du territoire, (d) l'impact des solutions sur l'autonomie du secteur agricole et du territoire et (e) la capacité à mobiliser les compétences internes au collectif. Cette sélection doit notamment apporter une opérationnalité favorisant ainsi l'implication des porteurs d'enjeux locaux. L'approche à des échelles inférieures s'avère notamment nécessaire quand les niveaux de gestion légitimes, notamment politique, s'opèrent à ces échelles (compétences de gestion et de décision affectées aux collectivités territoriales par exemple).

(iii) Le traitement des cas d'étude choisis dans l'étape 2, motivée plus particulièrement par la recherche de projets de symbiose bioéconomique territoriale (Bijon et al., 2020). L'utilisation d'outils de modélisation participative, notamment spatialisés, revêt un intérêt particulier pour y parvenir (Etienne, 2009).

(iv) L'évaluation multicritère et intégrée des solutions émergeant des différents cas d'étude. Celle-ci consiste à évaluer conjointement et à l'échelle du territoire étudiée, les différents projets locaux identifiés dans l'étape 3. Une approche de modélisation multicritère (technique, environnement, économie, social) et intégrée, incomplète dans GABiR notamment sur les aspects économiques, s'avèrerait particulièrement utile. Elle pourrait s'appuyer notamment sur le schéma de flux construit en étape 1 . Elle permettrait d'analyser les différentes interactions entre ces projets locaux, de type synergie, antagonisme ou les effets de compétition sur les ressources, au regard des différents enjeux du territoire.

(v) La concertation multi-acteurs et la définition d'un projet territorial commun. Cette définition devrait s'appuyer sur un consensus, ou a minima sur l'adhésion du plus grand nombre de représentants des différents acteurs, élaboré à partir d'une connaissance égale des éléments mis en lumière dans l'étape 4. II ne s'agit pas ici d'extrapoler les solutions locales à des échelles supérieures mais bien de voir comment le panel de solutions locales mises en lumière dans les étapes précédentes, et leur intégration, nourrissent ce projet territorial. Il n'est pas exclu toutefois, que ces réflexions mettent en évidence, dans un principe de subsidiarité, des problématiques qui ne peuvent être résolues qu'à cette échelle supérieure. Elles pourront ainsi compléter ce projet territorial mais poseront alors à nouveau les questions d'acteurs à impliquer et de solutions à envisager pour les résoudre, et faire ainsi l'objet de nouvelles études, nécessitant des retours à des étapes antérieures. Le cadre méthodologique prévoit donc de nombreuses itérations permettant d'aboutir à un projet territorial stabilisé et partagé par les différents acteurs du territoire.

\section{Conclusions et perspectives}

Dans les attentes sociétales et d'expression politique de relocalisation de l'agriculture, mais aussi de la place prépondérante du secteur agricole dans les dynamiques d'économie circulaire, la mise en œuvre de démarches cliniques à l'échelle territoriale devient indispensable. Si l'approche menée dans le projet GABiR a effectivement abouti à l'identification de solutions construite à la suite de 
concertations avec des mises en action plus ou moins avancées selon les cas d'étude, une extrapolation de ces dernières à l'ensemble de l'île semble difficile du fait de la diversité des contextes locaux et de la multiplicité des enjeux régionaux.

C'est en ce sens que de nouveaux travaux visant à gérer les synergies et les compromis à l'échelle de l'île sont en cours (Vayssières et Bravin, 2020), en s'appuyant encore sur les outils de modélisation spatiale. Les résultats obtenus pourraient ainsi permettre de dégager des pistes pour nourrir la quatrième et la cinquième étape du cadre générique proposé, qui n'ont pu être menées dans le projet, et permettant ainsi de « boucler » la démarche.

Ce retour d'expériences pourra également contribuer à améliorer le cadre générique d'accompagnement d'initiatives d'économie circulaire impliquant l'agriculture proposé ici. Il serait également utile de le confronter à d'autres démarches initiées dans des projets visant des objectifs similaires, et à d'autres contextes, y compris non-insulaires qui cristallisent de fait moins les enjeux d'autonomie et posent des questions plus complexes de délimitation du territoire étudié. En particulier, les étapes d'intégration des solutions locales pour la construction d'un projet territorial, et potentiellement la mise en lumière de problématiques qui ne pourraient être résolues qu'à des échelles supérieures pourrait faire émerger de nouvelles questions de recherche. Dans le cas de GABiR par exemple, la mise en cohérence des différents cas d'étude fait apparaitre des risques de compétition autour des différents usages des biomasses. Ainsi, un besoin d'accompagnement par la Recherche sur les décisions politiques prises par la Région et le Département, en particulier pour la hiérarchie et le soutien des initiatives en cours ou à venir, émerge.

Toutefois, si la modélisation, en particulier spatialisée, se révèle un outil puissant pour favoriser cette démarche, il apparait également que celle-ci ne peut être mobilisée sans la maîtrise d'outils d'animation et de concertation collective. Ce constat pointe donc la nécessité d'intégrer la multiplicité des métiers d'agronomes, depuis l'agronome des cultures ou le zootechnicien jusqu'à la modélisation, en passant par l'agronome «médiateur ».

\section{Remerciements}

Ces travaux ont été menés dans le cadre du projet GABiR, financé par le Ministère de l'Agriculture et de l'Alimentation au titre du compte d'affectation spéciale « Développement agricole et rural » (CASDAR) dans le cadre de l'appel à projet Innovation et Partenariat 2016.

\section{Références}

Bijon, N., Wassenaar, T., Junqua, G., Dechesne, M., 2020. Définir le Système-Territoire pour une Symbiose Territoriale bioéconomique. T2020 - Transitions écologiques en transactions et actions, Juin 2020, Toulouse, France.

Bonaudo, T., Domingues, J.P., Tichit, M., Hauber Gameiro, A., 2016. Intérêts et limites de la méthode du métabolisme territorial pour analyser les flux de matière et d'énergie dans les territoires d'élevage. 23ème 3R. 7-8/12/2016, Paris, France.

BRLi, 2017. Etude prospective sur la ressource fourragère. BRL ingénierie, rapport interne. 87 p.

Cerf, M., Parnaudeau, V., Reau, R., 2019. Vers un diagnostic agronomique stratégique traitant de questions agro-environnementales. Agronomie, Environnement \& Sociétés 9(2), $27-37$.

Cornec, R., Mir, C., 2018. Gestion des déchets sur l'île de la Réunion. Ministère De La Transition Écologique et Solidaire, Rapport n012231-01. $92 \mathrm{p}$.

DellaCroce, C., Tironi, Y., 2018. Le diagnostic participatif : un outil pour pratiquer la recherche action. Animation, territoires et pratiques socioculturelles 14, 1-14.

Darras, A., 2019. Exploration de scenarios de valorisation en agriculture des déchets verts urbains dans le sud de l'île de la Réunion. Mémoire de fin d'études. Institut des Régions Chaudes, Montpellier SupAgro, Montpellier. 
Degenne P., Lo Seen D., 2016. Ocelet: Simulating processes of landscape changes using interaction graphs. SoftwareX 5, 89-95.

Dumont, B., Fortun-Lamothe, L., Jouven, M., Thomas, M., Tichit, M., 2013. Prospects from agroecology and industrial ecology for animal production in the 21st century. Animal 7, 1028-1043.

Dupuy, S., Gaetano, R., Le Mezo, L., 2020. Mapping land cover on Reunion Island in 2017 using satellite imagery and geospatial ground data. Data in Brief 28, 104934.

Etienne, M., 2009. La modélisation d'accompagnement : une démarche participative en appui au développement durable. Editions Quae,Paris, France.

Gaffier, C., 2017. Analyse de la gestion des déchets verts urbains par un syndicat de traitement des déchets : stratégies actuelles et potentielles de valorisation dans le contexte réunionnais. Mémoire de fin d'études. AgroParisTech, Paris.

Grillot, M., Ruault, J.F., Torre, A., Bray, F., Malderieux, S., 2021. Le proto-métabolisme : approche du fonctionnement bioéconomique d'un territoire agricole. Economie Rurale 376, 55-75.

Jarry R., 2019. Modélisation des flux d'effluents d'élevages sur le territoire de Saint-Joseph, en lien avec la dynamique du bâti. Mémoire de stage de césure. AgroParisTech, Paris.

Kirchherr, J., Reike, D., Hekkert, M., 2017. Conceptualizing the circular economy: An analysis of 114 definitions. Resources, Conservation and Recycling 127, 221-232.

Kleinpeter, V., Vayssières, J., Alison, C., van de Kerchove, V., Degenne, P., Vigne, M., 2019. Inventaire et quantification des flux de biomasses locales valorisées ou valorisables en agriculture à La Réunion. Rapport technique issu du projet GABiR. 68 p.

Kockmann, F., Pouzet, A., Omon, B., Pavarano, L., Cerf, M., 2019. La démarche clinique en agronomie : sa mise en pratique entre conseiller et agriculteur. Agronomie, Environnement \& Sociétés 9(2), 15-26.

Lardon, S., 2005. Modélisation spatiale et approche agronomique. in Legay, J.M. (Ed.), L'interdisciplinarité vue et pratiquée par les chercheurs en sciences de la vie, Editions INRA, Paris, ,150169..

Leloup, F., 2010. Le développement territorial et les systèmes complexes : proposition d'un cadre analytique. Revue d'Économie Régionale \& Urbaine 4, 687-705.

Lorre, F., 2019. Evaluation du disponible fourrager à l'échelle de l'île de la Réunion et leviers pour une meilleure valorisation des surfaces fourragères. Mémoire de fin d'études. Ecole Supérieure d'Agricultures d'Angers, Angers.

Lorre, F., Magnier, J., Degenne, P., Mirales, M., Lurette, A., Vigne, M., Vayssieres, J., 2020. Appui de la modélisation spatialement explicite pour la mise en place d'une filière de fourrage structurée à l'échelle de l'île de La Réunion. 3R 2020.

MAAP, 2015. GIROVAR - Gestion Intégrée des Résidus Organiques par la Valorisation Agronomique à La Réunion. Compte-Rendu final du projet. Ministère de l'Alimentation, de l'Agriculture et de la Pêche, DGER/DI/BIPI, $\mathrm{n}^{\circ}$ du projet : 10004. $71 \mathrm{p}$.

Magnier, J., 2019. Evaluation de la consommation fourragère à la Réunion, construction et simulation de scénarios d'organisation d'une "filière fourrages ". Mémoire de fin d'études. Ecole Supérieure d'Agricultures d'Angers, Angers.

Madelrieux, S., Buclet, N., Lescoat, P., Moraine, M., 2017. Écologie et économie des interactions entre filières agricoles et territoire : quels concepts et cadre d'analyse? Cahiers Agricultures 26, 24001.

Ministère de la Transition Ecologique, 2018. Feuille de route Économie circulaire : 50 mesures pour une économie 100\% circulaire. 46 p. 
Moine, A., 2006. Le territoire comme un système complexe : un concept opératoire pour l'aménagement et la géographie. L'Espace géographique 2, 115-132.

Sébillote, M., 2002. Les trois métiers des agronomes. In Prevost, P. (Ed.), Agronomes et territoires deuxième édition des Entretiens du Pradel, L'Harmattan, Paris, 479-497.

Sébillote, M., 2006. Penser et agir en agronome In Doré et al. (Eds), L'agronomie d'aujourd'hui, Versailles, Quae, 1-21.

Taillandier, P., Gaudou, B., Grignard, A., Huynh, Q., Marilleau, N., Caillou, P., Philippon D., Drogoul, A.,2018. Building, composing and experimenting complex spatial models with the GAMA platform. Geoinformatica 23, 299-322.

Vall, E., Chia, E., 2014. Co-construire l'innovation : la recherche-action en partenariat. in Sourrisseau, J.M. (Ed.), Agricultures familiales et mondes à venir, Versailles, Quae. 239-255.

Vayssières, J., Bravin, M., 2020. L'agriculture au coeur de l'économie circulaire. Agronews, Edition Réunion-Mayotte / Océan Indien - Hors Série Avril 2020. 20 p.

Vinck D., 1999. Les objets intermédiaires dans les réseaux de coopération scientifique. Contribution à la prise en compte des objets dans les dynamiques sociales. Revue Française de Sociologie 40, 385-414.

Wassenaar, T., 2015. Reconsidering industrial metabolism: from analogy to denoting actuality. Journal of Industrial Ecology 19, 715-727.

Wassenaar, T., Queste, J., Paillat, J. M., Saint Macary, H., 2015. La co-construction de filières de recyclage de résidus organiques à la Réunion. Innovations Agronomiques 43, 161-175. mentionner son titre, le nom de tous les auteurs, la mention de sa publication dans la revue AE\&S et de son URL, ainsi que la date de publication. 